\title{
Pedestrian Physics Walks Away with Two Ig Nobels
}

\author{
This year's Ig Nobel prize winners include two groups of physicists \\ studying how humans avoid-and sometimes don't avoid-collisions \\ while walking. \\ By Michael Schirber
}

7 he Ig Nobel prizes have once again provided a light-hearted look at recent scientific achievements that "make people laugh, then think." This year, two of the prizes went to physicists studying collision avoidance among pedestrians. The award pair highlights the growing interest among physicists in probing the underlying "forces" that control crowd behavior.

The pedestrian physics work and eight other scientific efforts-including a study of cat-human communication, a microbial analysis of discarded chewing gum, and a survey of the emotions of movie watchers based on the chemicals they exhale-were recognized last week during the 31st First Annual Ig Nobel Prize ceremony. This was the second time that the festivities were conducted online because of the ongoing

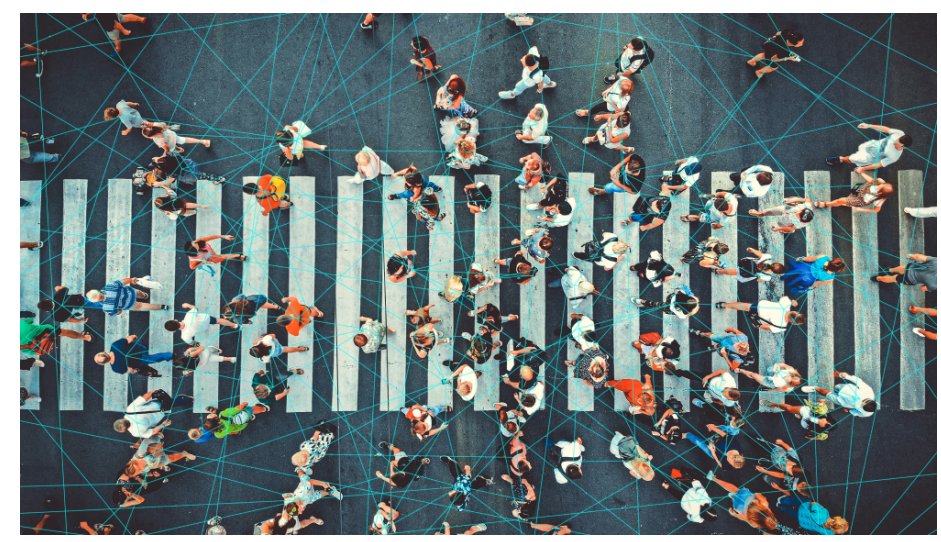

Physicists track pedestrian movements to understand how people avoid colliding with each other.

Credit: Dmytro/stock.adobe.com
COVID-19 pandemic.

Marc Abrahams-the editor of the magazine Annals of Improbable Research (which organizes the prize)-ran the zany video conference, with traditional features such as a paper-airplane toss and a science-themed opera. The awards-a build-your-own paper trophy and a ten-trillion-dollar bill in Zimbabwean currency-were handed out virtually by genuine Nobel laureates, including Carl Wieman and Eric Cornell (who shared the physics Nobel in 2001).

The physics prize went to Alessandro Corbetta from Eindhoven University of Technology in the Netherlands and his colleagues for their study of pedestrian dynamics in a train station. "We were ignobly surprised by the announcement," Corbetta joked in a follow-up interview. He said that the Ig Nobel prize is an opportunity to make people aware that physics is not only for studying particles and planets.

"There are many unconventional systems-such as busy train stations-that can be understood by applying the language of physics," Corbetta said. By analyzing several million pedestrian trajectories, he and his colleagues found that crowd movement can be understood by assuming "social forces" that help prevent people from running into each other (Physics covered these results in 2018, see Synopsis: How Walkers Avoid Collisions).

However, another team of physicists found that our collision-avoidance abilities are not foolproof. Hisashi Murakami from the Kyoto Institute of Technology in Japan and his colleagues asked two groups of volunteers to walk past each 
other while some of the volunteers looked at their cellphones. "We wanted to interfere with the anticipatory ability of pedestrians," Murakami said.

As we all might guess, the pedestrians with cellphones often failed to notice their fellow walkers, leading to several run-ins and near collisions. But Murakami and his colleagues-who were awarded the Ig Nobel kinetics prize-found that even the pedestrians without cellphones had difficulty navigating through the crowd. The implication is that humans count on the awareness of others to coordinate their walking paths. "We discovered that mutual anticipation facilitates the orderly flow of movement in human crowds," Murakami said.

Why so much interest in pedestrian dynamics? "There is this idea that human behavior is not predictable," said Federico Toschi, one of Corbetta's co-winners from Eindhoven. But the statistical behavior of a large ensemble of individuals can be anticipated, he says. For example, scientists can measure how fast people walk on average in a well-lit hallway or outside in the rain. The data can then be used to design train stations, football stadiums, and other urban environments so that overcrowding can be avoided.

"We might also use our understanding to improve the quality of a museum visit by applying some stimuli so that people don't queue up in front of a painting," Toschi added. Rather than a museum guard, a subtle change in the lighting or a sound coming from the next room could provide a subliminal nudge, prodding you to move along and let others have a gaze at the Mona Lisa.

Just remember to turn your cellphone off.

Michael Schirber is a Corresponding Editor for Physics based in Lyon, France. 